\title{
TOWARDS A DEFINITION OF LOCAL STATIONARITY FOR GRAPH SIGNALS
}

\author{
Benjamin Girault, Shrikanth S. Narayanan, Antonio Ortega \\ University of Southern California, Los Angeles, CA 90089, USA
}

\begin{abstract}
In this paper, we extend the recent definition of graph stationarity into a definition of local stationarity. Doing so, we present a metric to assess local stationarity using projections on localized atoms on the graph. Energy of these projections defines the local power spectrum of the signal. We use this local power spectrum to characterize local stationarity and identify sources of non-stationarity through differences of local power spectrum. Finally, we take advantage of the knowledge of the spectrum of the atoms to give a new power spectrum estimator.
\end{abstract} nals

Index Terms - graph signal processing, stationary graph sig-

\section{INTRODUCTION}

The recently introduced extension of the definition of stationarity to the framework of graph signal processing allows us to study stochastic graph signals with respect to their spectral properties [1]. Applications such as Wiener filtering [2] or more generally power spectrum filtering through convolutive filters are made possible by those spectral properties. White and colored noises are also straightforward to generalize using the power spectrum of the graph signal, i.e. the expected squared modulus of its Fourier transform $\mathbb{E}\left[|\widehat{\mathbf{x}}(l)|^{2}\right]$. The definition gave rise to several methods to estimate this power spectrum, from the very simple direct estimator [1] to an extension of the Bartlett-Welch method to the graph framework [3].

However, despite its good interpretation in the graph Fourier domain, the interpretation of stationarity in the vertex domain remains elusive. Indeed, a graph signal is stationary if and only if its statistics are invariant through the graph translation operator [1,4], and this operator is well understood in the Fourier domain, but not so in the vertex domain, especially because of its complex nature. In [5], we addressed this question through energy bounds of the graph translation impulse response, and showed that it can be interpreted as a diffusion-like operator.

In this paper, we go a step further on the concept of stationarity by refining it through the definition of local stationarity. Such a definition finds its roots at the core of stationarity in the temporal domain where a temporal signal is stationary if it is statistically the same when viewed from any point of time. We extend this to the graph framework by requiring that a locally stationary graph signal be the same when viewed from any vertex. This gives rise to a clear interpretation of stationarity in the vertex domain, and more importantly, this allows to pinpoint the sources of non-stationarity. In the process, we also give a novel power spectrum estimator obtained from the local power spectrum.

The paper is organized as follows. sec. 2 recalls the classical framework of graph signal processing. sec. 3 gives the recent defi-

This work was supported in part by NSF under grants CCF-1410009, CCF-1527874, CCF-1029373. nition of graph stationarity, while sec. 4 gives the new definition of local stationarity and discuss how to formalize it. Finally, experiments are carried out in sec. 5 .

\section{GRAPH SIGNAL PROCESSING}

We first state the concepts from graph signal processing that are used in this paper. Let $\mathcal{G}=(V, E)$ be a graph with $V=\{1, \ldots, N\}$ the set of vertices and $E \in V \times V$ the set of edges between vertices. We focus here on undirected graphs: if $(i j) \in E$, then $(j i) \in E$. Let $A$ be the weighted adjacency matrix with $a_{i j}$ the weight of the edge $i j$, or 0 if no such edge exists. Let $D=\operatorname{diag}\left(d_{1}, \ldots, d_{N}\right)$ be the degree matrix with $d_{i}=\sum_{j} a_{i j}$ the degree of vertex $i$. Let $L=D-A$ be the Laplacian matrix.

A graph signal $x=\left(x_{1}, \ldots, x_{N}\right)^{T}$ maps vertices to scalar values (real or complex). The graph Fourier transform (GFT) of $x$ is then defined as the projection onto the eigenvectors $\chi_{l}$ of $L$, with $L \chi_{l}=\lambda_{l} \chi_{l}$, and such that $\widehat{x}(l)=\left\langle x, \chi_{l}\right\rangle=\sum_{i} x_{i} \chi_{l}^{*}(i)$ [6]. Noting $F$ the matrix of the GFT, we have $F^{*}=\left[\chi_{0} \ldots \chi_{N-1}\right]$ and $\widehat{x}=F x$ and $x=F^{*} \widehat{x}$ since $F$ is unitary [2]. Note that the uniqueness of the matrix $F$ is not guaranteed when there is a non unique eigenvalue $\lambda_{l}=\lambda_{l+1} \cdot \lambda_{l}=\chi_{l}^{*} L \chi_{l}$ represents the variation of the Fourier mode $\chi_{l}$ on the graph and ranges from $\lambda_{0}=0$ to $\lambda_{N-1} \leq \rho_{\mathcal{G}} \leq 2 d_{\text {max }}$, with $\rho_{\mathcal{G}}$ a carefully chosen upper bound on $\lambda_{N-1}[4]$.

In this paper, boldface is used for stochastic variables. For example, $\mathbf{x}=\left(\mathbf{x}_{1}, \ldots, \mathbf{x}_{N}\right)^{T}$ is a stochastic graph signal.

\section{STATIONARY GRAPH SIGNAL: GLOBAL DEFINITION}

The original definition of stationarity as stated in [1,2] involves extending the time shift operator to the graph framework. This in turns allows to define graph signal stationarity as a statistical invariance through the graph shift. We use the graph translation as a generalization of the time shift to graph signals [4]. This operator amounts to a phase shift of the Fourier modes: $T_{\mathcal{G}} \chi_{l}=\exp \left(-\imath \pi \sqrt{\lambda_{l} / \rho_{\mathcal{G}}}\right) \chi_{l}$.

Definition 1 (Graph Translation [4]).

$$
T_{\mathcal{G}}:=\exp \left(-\imath \pi \sqrt{\frac{L}{\rho_{\mathcal{G}}}}\right) .
$$

We obtain then definitions of strict and wide sense stationarity:

Definition 2 (Strict Sense Stationary [1]). A stochastic signal $\mathbf{x}$ on the graph $\mathcal{G}$ is Strict-Sense Stationary (SSS) if and only if:

$$
\mathbf{x} \stackrel{d}{=} T_{\mathcal{G}} \mathbf{x},
$$

where $\stackrel{d}{=}$ stands for equality of probability distributions.

Definition 3 (Wide-Sense Stationary [1]). A stochastic signal $\mathbf{x}$ on the graph $\mathcal{G}$ is Wide-Sense Stationary (WSS) if and only if:

$$
\mu_{\mathbf{x}}:=\mathbb{E}[\mathbf{x}]=\mathbb{E}\left[T_{\mathcal{G}} \mathbf{x}\right]
$$




$$
R_{\mathbf{x}}:=\mathbb{E}\left[\mathbf{x x}^{*}\right]=\mathbb{E}\left[\left(T_{\mathcal{G}} \mathbf{x}\right)\left(T_{\mathcal{G}} \mathbf{x}\right)^{*}\right] .
$$

In Def. 3, $\mu_{\mathbf{x}}$ is the mean of the graph signal $\mathbf{x}$ and $R_{\mathbf{x}}$ is the autocorrelation matrix. Def. 3 states the invariance of these two quantities under the application of the graph translation to the stochastic graph signal $\mathbf{x}$. This definition has an interesting interpretation in the Fourier domain:

Property 1 (Spectral Characterization [1]). A stochastic signal $\mathbf{x}$ on the graph $\mathcal{G}$ is WSS if and only if

$$
\begin{aligned}
\widehat{\mu_{\mathbf{x}}}(l)=\mathbb{E}[\widehat{\mathbf{x}}(l)] & =0 & & \text { if } \lambda_{l}>0 \\
\Gamma_{\mathbf{x}}(k, l)=\mathbb{E}\left[\widehat{\mathbf{x}}(k) \widehat{\mathbf{x}}^{*}(l)\right] & =0 & & \text { if } \lambda_{k} \neq \lambda_{l} .
\end{aligned}
$$

In other words, Def. 3 is equivalent to the first moment $\mu_{\mathrm{x}}$ of $\mathbf{x}$ being a DC signal, i.e. a graph signal whose Fourier transform is only non-zero on the first Fourier mode, and the spectral autocorrelation matrix $\Gamma_{\mathrm{x}}=F R_{\mathrm{x}} F^{*}$ being block diagonal, with blocks corresponding to equal eigenvalues. We denote $\widehat{\gamma_{\mathbf{x}}}(l)=\Gamma_{\mathbf{x}}(l, l)$ the power spectrum of $\mathbf{x}$. Furthermore, assuming that all eigenvalues are distinct ${ }^{1}$, the matrix $\Gamma_{\mathbf{x}}$ is diagonal and we can $[3]^{2}$, and the inverse GFT $\gamma_{\mathbf{x}}$ of $\widehat{\gamma_{\mathbf{x}}}$ to characterize WSS graph signals:

Property 2 (Localization Characterization [3]). Assuming uniqueness of the eigenvalues of $L$, a stochastic signal $\mathbf{x}$ on the graph $\mathcal{G}$ is WSS if and only if $\mu_{\mathbf{x}}=\mathbb{E}[\widehat{\mathbf{x}}(0)] \chi_{0}$ and:

$$
\forall i, j \in V, R_{\mathbf{x}}(i, j)=\left(T_{j} \gamma_{\mathbf{x}}\right)_{i} .
$$

In Property 2, the operator $T_{j}$ is the localization operator verifying $T_{j} x=x * \delta_{j}=\sum_{l} \widehat{x}(l) \widehat{\delta}_{j}(l)$ with $*$ the generalized convolution operator [7]. $\gamma_{\mathbf{x}}$ is the autocorrelation operator of the stochastic graph signal $\mathbf{x}$. The advantage of this formulation is that it can lead to a definition of local stationarity. Indeed, it is well known that when all $\widehat{\gamma_{x}}(l)$ can be written as a polynomial of $\lambda_{l}$, then $T_{j} \gamma_{x}$ is localized around $j$, with the localization being tighter if the polynomial is of lower order [5]. In other words, if said polynomial is of low order, correlations extend only to the vicinity of a vertex. In that case, studying those vertices is enough to draw conclusions on the stationarity, and the power spectrum, of a graph signal. We formalize this into the notion of local stationarity in the rest of this paper.

\section{LOCAL STATIONARITY}

We give in this section a first informal definition of local stationarity, and develop a framework to illustrate this concept. Additionally, we give a sensible interpretation of local stationarity in the vertex domain.

\subsection{Definition and Premises}

We define local stationarity as the property that a signal "looks the same" in any neighborhood. More precisely, given a neighborhood span corresponding to how far from a vertex we observe a signal, then for any two vertices, the signal on these two neighborhoods shall be statistically the same. For example, this can be the $k$-hop neighborhood. Our goal is to show how to formalize local stationarity and apply this formalism to synthetic and real data. Doing so, we devise a new method to estimate the (global) power spectrum from this formalism.

\footnotetext{
${ }^{1}$ The more complex case of a graph with multiple eigenvalues will be covered in a future paper.

${ }^{2}$ We swapped $i$ and $j$ compared to the reference to account for complex graph signals.
}

This informal definition of local stationarity finds its premises in the temporal framework. Indeed, we know that a temporal WSS signal can be split into many smaller signals of equal length with each of them being statistically the same. In [8], the author leverages this property by carefully selecting windows of compact temporal support and optimal frequency concentration such that projection of the signal on those windows can be used to obtain a good estimator of the power spectrum density.

However, one crucial difference between graph and temporal frameworks is that graph Fourier modes can be highly localized [9, 10], whereas classical Fourier modes are delocalized. This has an adverse impact on the definition of local stationarity. The challenge is that given a Fourier mode localized on vertex $i$, and given two windows about vertex one around vertex $i$ and the other around another vertex $j$, which is not close to vertex $i$, then this Fourier mode may have very different values in the two windows, with most of the energy in the window centered in $i$, and no energy around $j$. We see with this example, that contrary to the global definition of stationarity, there are restrictions to the power spectrum of a locally stationary graph signal. For instance, the example above shows that the signal whose power spectrum is a delta centered on that Fourier mode is not locally stationary. In [3], the authors made an assumption of smoothness of the power spectrum that can be interpreted with this remark. However, the windows they use to perform power spectrum estimation are not localized such that this approach cannot be used to define local stationarity.

\subsection{Our Framework}

We now study one path towards a formal definition of local stationarity for graph signals. When introducing local stationarity, we have used the so-called $k$-hop neighborhood. This gives a first definition of windows on the graph. However, this simple definition has two drawbacks: the window edges are sharp, and the weights on the graph are not taken into account. Several approaches can be devised to address this point, from refining the k-hop neighborhood (e.g. using shortest-path or diffusion distances), or using the GSP toolbox.

In this paper, we choose the later and use as windows a set of localized atoms $\left\{g_{i, m}\right\}_{i, m}$ with $i$ the vertex on which the atom is centered, and $m$ corresponding to the span of the window on the graph. The associated decomposition of a signal $x$ onto this set of atoms is given by $w_{x}(i, m)=\left\langle x, g_{i, m}\right\rangle$. We then have:

Definition 4 (Local Power Spectrum). The local power spectrum of a stochatic graph signal $\mathbf{x}$ about vertex $i$ at scale $m$ is given by:

$$
S_{\mathbf{x}}(i, m):=\mathbb{E}\left[\left|w_{\mathbf{x}}(i, m)\right|^{2}\right]
$$

Note that this quantity is not strictly a power spectrum since the atoms should not be localized in the frequency domain to account for localized Fourier modes, as stated before. Nevertheless, we will see that they can be used to perform spectrum estimation.

We are left with the definition of the atoms $g_{i, m}$. As discussed before, these atoms should be more or less localized on $i$ depending on $m$. We arbitrarily choose to increase localization with $m$. One way to achieve localization is using the localization operator of [7] applied to a signal $g_{m}$ with smooth GFT. Indeed, if $\widehat{g_{m}}(l)$ can be written as a polynomial of $\lambda_{l}$ of low order (or can be well approximated with a low order polynomial), then the atom $T_{i} g_{m}$ is localized about $i$, depending on the order of the polynomial [5].

We are left with choosing the set of signals $\left\{g_{m}\right\}_{m}$. Formal definition of such a set will be the subject of a future paper, and we now explore a few possibilities from the literature, and one simple alternative. A review of the literature on tight frames for graph signals 
can be found in [11], where the authors explore several definitions of $g_{m}$. However, most of those definitions do not yield good localization properties (i.e. high order polynomials are required in the definition of $g_{m}$ from $\left.\left\{\lambda_{l}\right\}\right)$. This is the same for the definition used in [3] where the authors define $\widehat{g_{m}}(l)=g\left(\lambda_{l}-m \tau\right)$ where $g\left(\lambda_{l}\right)$ can be approximated with a low order polynomial, but its translations in $g_{m}$ cannot. One definition of $g_{m}$ in [11] does have the required localization properties: the Spectral Graph Wavelet Transform (SGWT) of [12].

In this paper, we focus on this transform, and on a new waveletlike decomposition having the theoretical advantage of not being biased in the Fourier domain. This new basis, which will be called "Expo", is built from the signals $g_{m}$ :

$$
\widehat{g_{m}}(l)= \begin{cases}\exp \left(-\kappa \lambda_{l}\right) & \text { if } m=0 \\ \exp \left(-\kappa \frac{\lambda_{l}}{2^{m}}\right)-\exp \left(-\kappa \frac{\lambda_{l}}{2^{m-1}}\right) & \text { otherwise, }\end{cases}
$$

with $\kappa$ a free parameter. The advantage of this decomposition is the property that $\sum_{m} \widehat{g_{m}}=1$ such that there is no bias towards any graph frequency. The value of $\kappa$ should then be chosen such that when considering the signals $g_{m}$ with $m \leq M$, their summed spectrum spans the graph frequencies well enough. Example of frequency responses are shown on Fig. 2 for a particular graph, with $M=15$. We can show that the atoms constructed in this manner are approximately local using [5], and that the locality is tighter for larger values of $m$.

\subsection{Theoretical Properties}

We use the local power spectrum $S_{\mathbf{x}}(i, m)$ in two different ways. First studying its variation with $i$ gives information on whether the graph signal is locally stationary. Note that due to the atoms being of different energy (see Fig. 2), the local spectrum needs to be rescaled to remove the bias of this energy (first row of Fig. 3): $S_{\mathbf{x}}(i, m) /\left|g_{i, m}\right|_{2}^{2}$. To illustrate the difficulties of finding a good set of atoms, we study the local power spectrum of a WSS graph signal:

$$
S_{\mathbf{x}}(i, m)=\sum_{l} \widehat{\gamma_{\mathbf{x}}}(l)\left|\widehat{g_{m}}(l)\right|^{2}\left|\chi_{l}(i)\right|^{2}
$$

We see here that having $S_{\mathbf{x}}(i, m)$ independent of $i$ would require $\left|\chi_{l}(i)\right|$ independent of $i$, which is not the case, such that global stationarity does not imply local stationarity. This is an illustration of the earlier point stating that the power spectrum of locally stationary graph signal cannot be arbitrary due to the localization of some Fourier modes.

The second use of the local power spectrum is to perform an estimation of the global spectrum when the signal is WSS. Indeed, let $\mathbf{x}$ be a WSS graph signal. We can show that:

$$
S_{\mathbf{x}}(m):=\sum_{i} S_{\mathbf{x}}(i, m)=\sum_{l} \widehat{\gamma_{\mathbf{x}}}(l)\left|\widehat{g_{m}}(l)\right|^{2} .
$$

This relation can be written in matrix form as $H \widehat{\gamma_{\mathbf{x}}}=S_{\mathbf{x}}$, with $S_{\mathbf{x}}$ the column vector of $S_{\mathbf{x}}(m)$ and $H$ the rectangular matrix with $H_{m, l}=\left|\widehat{g_{m}}(l)\right|^{2}$. Using a least-square estimator yields an estimation of $\widehat{\gamma_{\mathbf{x}}}$ from the knowledge of $S_{\mathbf{x}}$.

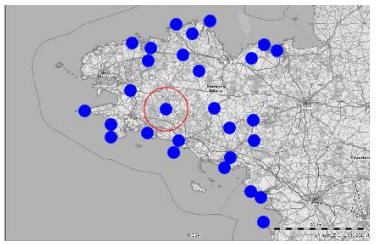

Fig. 1: Molène graph. Circled in red: non-stationarity identified in Fig. 5.
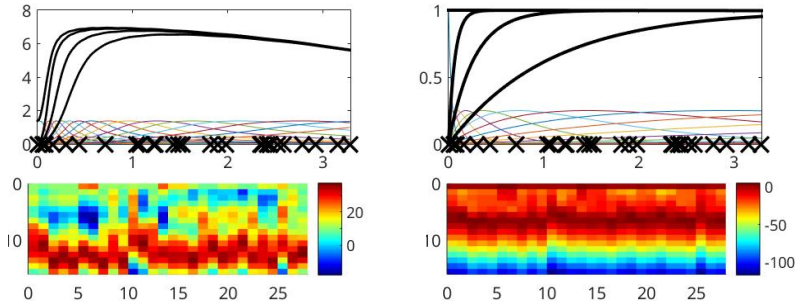

Fig. 2: Top: Spectra of $g_{m}$ for $m \in\{0, \ldots, M=15\}$ (in dB). Bold curves: sum of spectra $\left(M_{\min } \in\{0,2,4,6\}\right)$. Crosses: eigenvalues of the Molène graph. Bottom: Energy of the atoms. Left: SGWT. Right: Expo.

We use also a similar estimator without the coarsest scales, i.e. only the atoms with $m \geq M_{\min }$, the rationale being that if we can infer the global power spectrum from local quantities, then we can easily perform distributed stochastic graph signal processing. Mathematically, the least-square estimator is performed with the last elements of $S_{\mathrm{x}}$ and the last rows of $H$ above.

\section{EXPERIMENTS}

In this section, we experiment with the framework described above on a geographical graph. We show three uses of the local power spectrum: non-stationarity detection, power spectrum estimation, and power spectrum approximation using the finer windows (atoms associated to larger values of $m$ ).

\subsection{Graph}

The graph we use is the Molène graph $[1,2,13]$. Its vertices are 28 weather stations in the Brittany region, France. The dataset has been published by the French national weather agency ${ }^{3}$, and comes with hourly readings of the weather stations over the month of January 2014 (744 readings). We build the edges of the graph using a Gaussian kernel of the geographical distance between vertices as edge weights $\left(a_{i j}=\exp \left(-d_{i j}^{2} /\left(2 \sigma^{2}\right)\right)\right.$, with $\left.\sigma^{2}=5.10^{9}\right)$, and remove the edges corresponding to distances greater than $96 \mathrm{~km}$ $\left(a_{i j}<10^{-4}\right)$. The graph is shown on Fig. 1 .

We build then the set of atoms from the SGWT and the Expo schemes described in the previous section. Fig. 2 shows the spectrum of the signals $g_{m}$ for both decompositions, along with the spectrum of their sum and their truncated sum (where coarsest atoms are removed). We remark that the spectrum of the sum is flat for the Expo decomposition, but its truncated sum influences high frequencies more than the SGWT, meaning that SGWT should give more stable results in the high frequency spectrum when approximating the power spectrum from a truncated decomposition. Notice also that the DC-component is only found for $m=0$, and as soon as $M_{\min }>0$, this power of the DC-component cannot be recovered by the estimator.

Fig. 2 also shows the energy of the atoms. The energy of the SGWT atoms is larger for finer scales ( $m$ large) whereas the Expo decomposition has very low energy for those scales, suggesting again less bias when approximating the power spectrum from a truncated decomposition for SGWT.

\subsection{Synthetic Data}

Before studying real (weather) data, we begin with synthetic data for which we can choose a ground truth for the power spectrum. To

\footnotetext{
${ }^{3}$ Published under the title "Données horaires des 55 stations terrestres de la zone Large Molène sur un mois" on http: / / data.gouv. fr.
} 

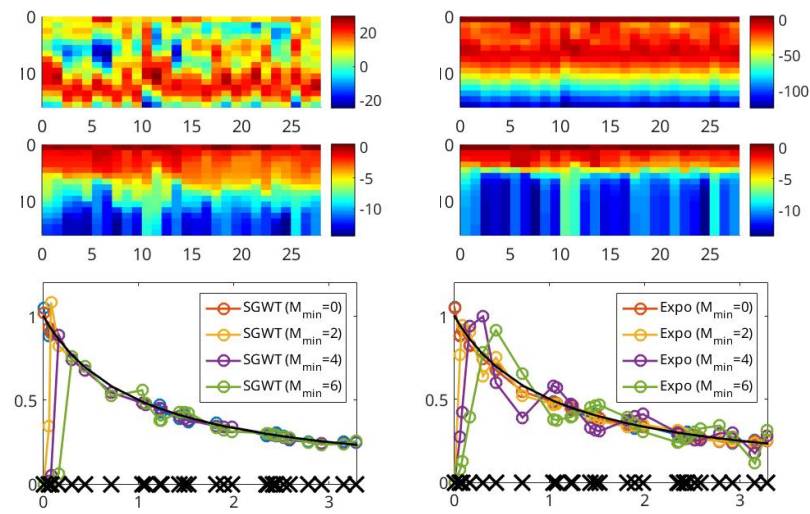

Fig. 3: Local power spectrum (in $\mathrm{dB}$ ) of 744 realizations of the GMRF model (with $a=1$ ), without (top) and with (middle) normalization. Bottom: PSD estimate with only the local power spectrum verifying $m \geq M_{\min }$ (Blue: Simple, Black: Ground Truth). Left: SGWT. Right: Expo.

that end, we choose a simple GMRF model with power spectrum $\widehat{\gamma_{\mathbf{x}}}(l)=\left(a+\lambda_{l}\right)^{-1}[14]$. This power spectrum is smooth for high frequencies (almost flat), hence interesting for the study of local stationarity. We generate 744 realizations of this model, and estimate the local power spectrum by averaging $\left|w_{\mathbf{x}}(i, m)\right|^{2}$.

As shown on Fig. 3, the local power spectrum without normalization is not very informative due to the atoms being of different energy (first row), while after normalization, we clearly see similarities between local power spectrum of vertices with both the SGWT and the Expo decompositions.

Comparison of various approaches to spectrum estimation is shown on Fig. 4. The Simple estimator refers to the estimator computing the Fourier transform of each realization and performing the mean power spectrum, Perraudin et al. refers to the approach of [3]. All four of them perform very well on the GMRF model. As expected, the simple estimator has more variance than the other.

Finally, the last row of Fig. 3 shows how the estimator behaves when the decomposition is truncated. As expected, the SGWT yields a more stable estimator when only the lowest scales are missing.

\subsection{Real Data}

The dataset includes also weather readings, among which the temperature is of particular interest to us. We preprocess the data according to [2] to remove the temporal dependency of the readings in order to assume ergodicity and obtain relevant statistical estimators.

Fig. 5 (top row) shows the resulting local power spectrum after normalization. A very interesting observation drawn from these local power spectrum estimates is the fact that one vertex is clearly different than the others with high spectral energy in finer scales: this vertex corresponds to the weather station of Guiscriff (vertex \#19 on Fig. 5, circled in red on Fig. 1). To assess whether this is a significant difference, we synthesize a WSS graph signal with the
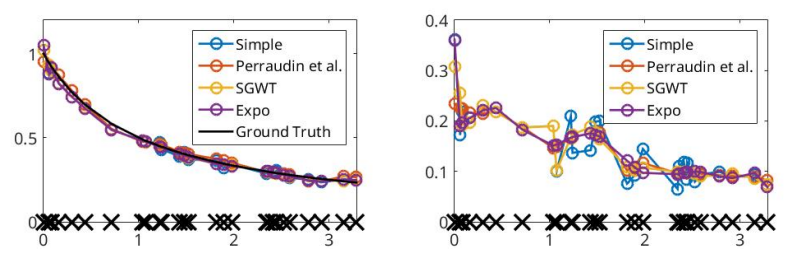

Fig. 4: Comparison of PSD estimators on the GMRF model (left) and the temperature dataset (right).
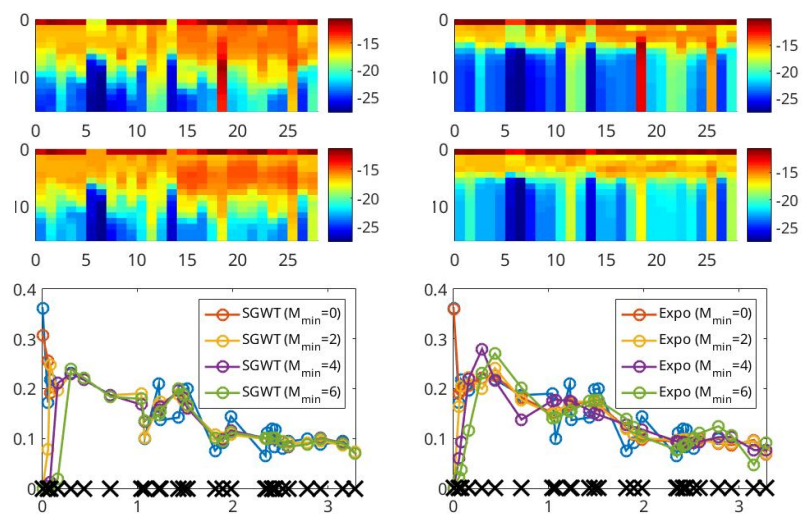

Fig. 5: Temperatures local PSD (in $\mathrm{dB}$ ) after preprocessing (top) and local PSD of a model (744 realization of a WSS graph signal with prescribed PSD equal to an estimate of the temperatures) (middle). Bottom: PSD estimate from truncated local PSD (Blue: Simple). Left: SGWT. Right: Expo.

same prescribed power spectrum (see [2] for details), and the same number of realizations (744). This yields the local power spectrum of Fig. 5 (middle row), where the local power spectrum of that vertex is not as dominant as in the temperature dataset. This difference can be explained from the data, where this particular weather station shows the highest short-time variation of temperatures $\left(+5.3^{\circ} \mathrm{C}\right.$, $+8.7^{\circ} \mathrm{C},+9.5^{\circ} \mathrm{C}$ in respectively 1,2 , and 3 hours), thus having the highest variance in the preprocessed data.

This is one very important use of the local power spectrum approach: identifying the sources of non-stationarity. Indeed, the global power spectrum alone cannot be used for that very purpose since the correlation matrix $R_{\mathrm{x}}$ of a WSS signal does not show any obvious structure, and the very simple non-stationary case of increasing the variance on one vertex does not lead to a spectral correlation matrix where we can identify the vertex (see [2] for details). Local power spectrum is therefore an essential tool to study non-stationarity.

Finally, power spectrum estimators yields slightly different results as shown on Fig. 4, with our estimators closer to the simple estimator in the low frequencies, and smoother in the high frequencies. This suggest that compared to [3], our method allows for more richness of the power spectrum in the lower part of the power spectrum. Finally, truncation of the local power spectrum yields similar results on the estimator with SGWT giving more stable estimates than Expo (see Fig. 5).

\section{CONCLUSIONS AND PERSPECTIVES}

This work on local stationarity yields highly interesting results, and in particular a definition of stationarity that is easy to interpret in the vertex domain. Moreover, sources of non-stationarity in the vertex domain become easier to pinpoint using local stationarity. Finally, we show that the local power spectrum we defined can be used to approximate the global power spectrum, and even approximate it without the use of the coarsest scales.

Numerous perspectives of local stationarity are being investigated, among which we can cite work on the signals $g_{m}$ to have better localization properties and/or power spectrum estimation, study of the local power spectrum, or how well this framework works for different classes of stochastic graph signals. Finally, we are looking into a local stationarity test extending the work of [15] based on our local power spectrum. 


\section{REFERENCES}

[1] Benjamin Girault, "Stationary Graph Signals using an Isometric Graph Translation," in Signal Processing Conference (EUSIPCO), 2015 Proceedings of the 23rd European. IEEE, 2015.

[2] Benjamin Girault, Signal Processing on Graphs - Contributions to an Emerging Field, Phd thesis, Ecole normale supérieure de lyon - ENS LYON, Dec. 2015.

[3] Nathanaël Perraudin and Pierre Vandergheynst, "Stationary signal processing on graphs," arXiv preprint arXiv:1601.02522, 2016.

[4] Benjamin Girault, Paulo Gonçalves, and Éric Fleury, “Translation on Graphs: An Isometric Shift Operator," Signal Processing Letters, IEEE, vol. 22, no. 12, pp. 2416-2420, Dec 2015.

[5] Benjamin Girault, Paulo Gonçalves, Shrikanth S. Narayanan, and Antonio Ortega, "Localization Bounds for the Graph Translation," in 2016 IEEE Global Conference on Signal and Information Processing, Washington D.C., USA, Dec 2016, accepted.

[6] David I. Shuman, Sunil K. Narang, Pascal Frossard, Antonio Ortega, and Pierre Vandergheynst, "The Emerging Field of Signal Processing on Graphs: Extending High-Dimensional Data Analysis to Networks and Other Irregular Domains.," IEEE Signal Processing Magazine, vol. 30, no. 3, pp. 83-98, 2013.

[7] David I. Shuman, Benjamin Ricaud, and Pierre Vandergheynst, "Vertex-frequency analysis on graphs," Applied and Computational Harmonic Analysis, vol. 40, no. 2, pp. 260-291, 2016.

[8] David J Thomson, "Spectrum estimation and harmonic analysis," Proceedings of the IEEE, vol. 70, no. 9, pp. 1055-1096, 1982.

[9] Bastien Pasdeloup, Réda Alami, Vincent Gripon, and Michael Rabbat, "Toward An Uncertainty Principle For Weighted Graphs," in Signal Processing Conference (EUSIPCO), 2015 Proceedings of the 21 st European, Sept. 2015, pp. 1511-1515.

[10] Ameya Agaskar and Yue M. Lu, "A Spectral Graph Uncertainty Principle.," IEEE Transactions on Information Theory, vol. 59, no. 7, pp. 4338-4356, 2013.

[11] David I. Shuman, Christoph Wiesmeyr, Nicki Holighaus, and Pierre Vandergheynst, "Spectrum-Adapted Tight Graph Wavelet and Vertex-Frequency Frames," IEEE Trans. Signal Processing, vol. 63, no. 16, pp. 4223-4235, 2015.

[12] David K. Hammond, Pierre Vandergheynst, and Rémi Gribonval, "Wavelets on graphs via spectral graph theory," Applied and Computational Harmonic Analysis, vol. 30, no. 2, pp. 129-150, 2011.

[13] Benjamin Girault, Paulo Gonçalves, and Éric Fleury, "Signaux Stationnaires sur graphe : étude d'un cas réel," in Proc. of Gretsi 2015, Sept. 2015.

[14] Eduardo Pavez and Antonio Ortega, "Generalized Laplacian precision matrix estimation for graph signal processing," in 2016 IEEE International Conference on Acoustics, Speech and Signal Processing (ICASSP). IEEE, 2016, pp. 6350-6354.
[15] Pierre Borgnat, Patrick Flandrin, Paul Honeine, Cédric Richard, and Jun Xiao, "Testing stationarity with surrogates: a time-frequency approach.," IEEE Trans. Signal Processing, vol. 58, no. 7, pp. 3459-3470, 2010. 